\title{
Copépodes Harpacticoïdes cavernicoles de Bulgarie. 3. Description d'un nouveau copépode du genre Parastenocaris Kessler, 1913 de Bulgarie
}
A. Apostolov 1

Mots clés : Copépoda, Harpacticö̈da, espèce nouvelle, faune cavernicole, Bulgarie.

Description de Parastenocaris bulgarica n.sp., récoltée dans la grotte "Douchnica » au nord-ouest de la Bulgarie.

Cave-dwelling harpacticoid copepods from Bulgaria.

3. Description of a new copepod in the genus Parastenocaris Kessler, 1913 from Bulgaria

Keywords: Copepoda, Harpacticoida, new species, cave waters, Bulgaria.

A description is provided of Parastenocaris bulgarica n.sp., from the cave " Douchnica ", northwest Bulgaria.

La présente note est la suite de nos recherches sur la faune des Harpacticoïdes des eaux cavernicoles de Bulgarie (Apostolov 1986, 1989, 1991).

Durant les années 1988-1990, M. Ivan Pandursky, de l'Institut de Zoologie de Sofia, a recueilli une abondante collection de Copépodes Harpacticoïdes des eaux cavernicoles. Parmi les espèces récoltées, a été trouvé un représentant du genre Parastenocaris Kessler, 1913, nouveau pour la science, Parastenocaris bulgarica $\mathrm{n} . \mathrm{sp}$.

A ce jour, le genre Parastenocaris était représenté dans les eaux souterraines de Bulgarie par sept espèces : Parastenocaris brevipes Kessler, P. karamani Chappuis, $P$. mana Chappuis, $P$. panonica Török, $P$. phreatica Chappuis, $P$. bohemica Sterba, $P$. chappuisi Serban.

La nouvelle espèce Parastenocaris bulgarica n.sp. constitue done la huitième pour la faune des Harpacticoïdes des eaux cavernicoles de Bulgarie. Elle a été trouvée dans la grotte "Douchnika » près du village d'Iskretz au nord-ouest de la Bulgarie.

\section{Parastenocaris bulgarica n.sp.}

Matériel examiné : 3 femelles et 2 mâles.

1. Izgrev, B1.35, Bx.R, 8008 Burgas, Bulgarie.
Localité : Lac dans la grotte " Douchnika " près du village d'Iskretz (locus typicus), le 30 mai 1988 : 3 \% ; le 28 janvier $1989: 2$ o.

Holotype : 1 femelle.

Paratype : 1 femelle et 1 mâlę dans la collection de l'auteur.

\section{DIAGNOSE}

- Femelle

Antennule composée de sept articles. L'endopodite P2 uniarticulé portant deux cils dont un plus long que l'autre. Endopodites P3 et P4 de simples dards qui ne dépassent pas le premier article de l'exopodite. P5 bien développée, allongée, se terminant en une longue pointe aiguë, avec sur le bord externe une dent puis deux soies. Exopodites $\mathrm{P} 2$ et $\mathrm{P} 4$ triarticulés, exopodite P3 biarticulé. Rames furcales plus longues que larges, armées de deux soies apicales et deux soies latérales.

- Mâle

Surface du corps et bord postérieur des segments sans ornementation. Antenne préhensile. Endopodite P2 uniarticulé avec trois soies apicales. P3, patte copulatrice, sans endopodite visible. Le premier article de l'exopodite gros, allongé. Apophyse longue et se terminant en une pointe ; second article, le pouce, plus court que l'apophyse, en forme de dard. 
Endopodite P4, un petit dard qui n'atteint pas la moitié du premier article de l'exopodite, recourbé vers le côté interne. A sa base, du côté interne, deux petites épines recourbées vers le côté externe. P5 en forme d'une lamelle oblongue portant une épine apicale et deux autres plus petites subapicales au bord externe. Branches furcales comme chez la femelle.

\section{DESCRIPTION}

\section{- Femelle}

Longueur $0,34 \mathrm{~mm}$. Surface du corps et bord postérieur des segments sans ornementation. Opercule anal bien arrondi à son bord libre qui est glabre. Branches furcales 3,5 fois plus longues que larges. Bords parallèles ; bord interne glabre ; bord externe avec deux soies qui s'insèrent dans le dernier quart. Face dorsale avec la soie usuelle qui s'insère au début du dernier tiers. Partie distale avec deux soies apicales, l'externe longue, l'interne plus courte (Fig. $1,1)$.

Antennule (Fig. 1,3) : allongée, composée de sept articles, avec un bâtonnet sensitif $\mathrm{au} 4^{\mathrm{e}}$ article.

Antenne (Fig. 1,4) : coxa courte et nue. Endopodite uniarticulé.

Pièces buccales comme chez les autres espèces.

PI : l'exopodite triarticulé. Les articles sont sans soies internes. L'article médian sans épine externe. L'endopodite biarticulé. Le premier article atteint le milieu de l'article médian de l'exopodite et porte une soie interne. Le second article a deux soies apicales dont l'interne est la plus longue.

P2 (Fig, 1,5) : l'exopodite triarticulé : premier article avec une épine externe ; article médian sans épine externe ; article distal portant trois longues soies. L'endopodite est uniarticulé avec deux soies dont l'interne est plus longue.

P3 (Fig. 1,6) : l'exopodite biarticulé, avec une épine ext erne sur l'article basal et deux soies apicales sur l'article distal. L'endopodite en forme de simple dard.

P4 (Fig. 1,7) : l'exopodite triarticulé. Le premier article avec une longue épine externe qui atteint la fin de l'article médian ; le second article sans épine externe ; l'article distal porte deux soies dont l'interne est plus longue. L'endopodite en forme de simple dard.
P5 (Fig. 1,8) : bien développée, allongée, se terminant en une longue pointe aiguë. Sur le bord externe, une dent puis deux soies.

\section{- Mâle}

Longueur 0,31 mm. Branches furcales, opercule anal, $\mathrm{P} 1$, exopodites $\mathrm{P} 2$ et $\mathrm{P} 4$ comme chez la femelle et ne présentant aucun dimorphisme sexuel.

P2 (Fig. 1,9) : endopodite uniarticulé, allongé, portant à sa partie distale trois cils.

P3 : (Fig. 1,10) : sans endopodite visible. L'apophyse du premier article digitiforme ; second article qui a la forme d'une épine et ne dépasse pas l'extrémité de l'apophyse.

P4 (Fig. 1,11) : l'endopodite bien développé. C'est une grosse épine recourbée vers le côtè interne. A sa base, du côté interne, deux petites épines recourbées vers le côté externe.

P5 : en forme d'une lamelle, portant une épine apicale et deux autres plus petites subapicales au bord externe comme chez la femelle.

\section{POSITION SYSTEMATIQUE}

La nouvelle espèce correspond parfaitement à la diagnose du genre Parastenocaris Kessler. La forme et la structure de l'endopodite P4 du mâle provenant de Bulgarie ainsi que la présence d'épines à sa base conduisent à placer Parastenocaris bulgarica n.sp., dans le groupe « minuta » (Lang 1948).

Cette espèce ne peut être rapportée à aucune autre espèce du genre Parastenocaris. Seules quelques Parastenocaris, $P$. elegans, $P$. dubia, $P$, vicesima, $P$. pannonicus, $P$. minutissima, $P$. karamani, $P$. orcina, $P$. hippuris possèdent deux épines à la base de l'endopodite P4 du mâle.

$P$. vicesima et $P$. pannonicus se distinguent aisément de la nouvelle espèce par leurs branches furcales très courtes, par l'endopodite de la P3 et par l'endopodite de la P4 du mâle.

$P$. phreatica diffère de $P$. bulgarica n.sp. par la structure des branches furcales, par la forme et le nombre d'épines de la P5 du mâle.

$P$. elegans diffère de $P$. bulgarica n.sp. par la forme de la P4 du mâle et par la structure différente de la P5 du mâle.

$P$. minutissima et $\boldsymbol{P}$. karamani diffèrent de l'espèce bulgare par la forme et la structure de la P5 du mâle, par le rapport longueur/largeur des branches furcales et par les endopodites P3 et P4 du mâle. 


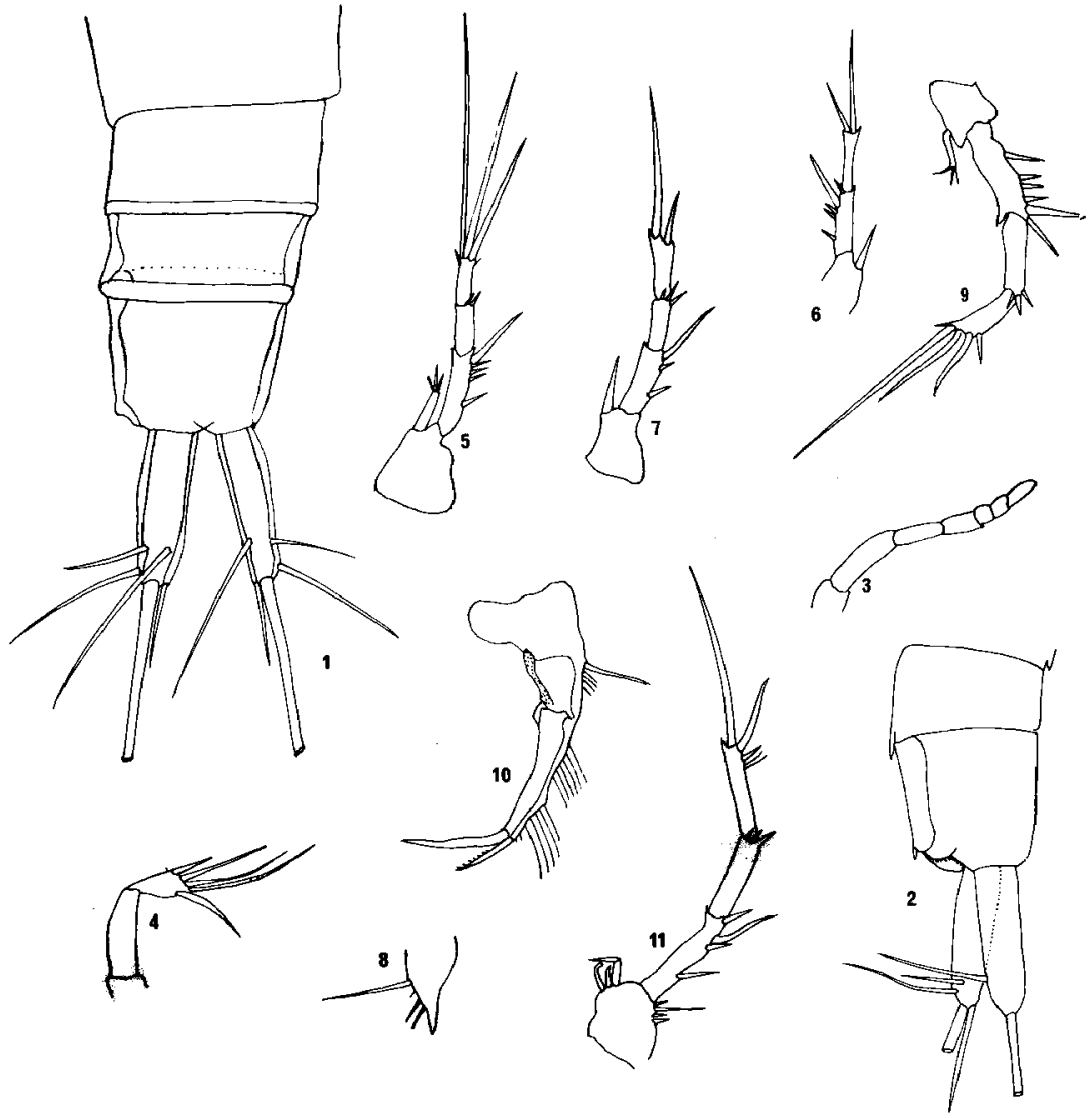

Fig. 1. Parastenocaris bulgarica n.sp.,$Q$ et $\sigma: 1$ - Furca, vue dorsale $\odot: 2$ w Furca, vue latérale $Q: 3$ - Antennule $Q: 4-$ Antenne

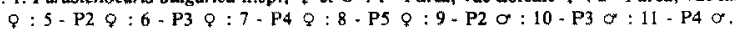


Les autres caractères distinctifs des exemplaires provenant de Bulgarie sont les suivants : branches furcales 3,5 fois plus longues que larges ; opercule anal arrondi ; apophyse de la P 3 du mâle bien développée ; endopodite P4 du mâle avec deux excroissances à sa base ; P5 de la femelle et du mâle avec deux courtes soies.

Enfin, les P1 et $P 2$ des mâles et des femelles, les $\mathrm{P} 3$ et $\mathrm{P} 4$ des femelles présentent des structures identiques à celles observées chez les autres espèces citées ci-dessus.

\section{Remerciements}

Je tiens à remercier M. Ivan Pandoursky de l'Institut de Zoologie de Sofia qui m'a confié l'étude des Harpacticoìdes des eaux cavernicoles de Bulgarie.
Travaux cités

Apostolov A. 1986. - Deux nouveaux copépodes souterrains de Bulgarie (Crustacea, Harpacticoïda), Boll. Mus. civ. Stor. nat. Verona, $13: 455-466$.

Apostolov A. 1989. - Description d'un nouveau représentant du genre Stygoelaphoidella Apostolov, 1985 (Copépoda, Harpacticoida) dans les eaux cavernicoles de Bulgarie. Annls Limnol. 25 (2) : $101-106$.

Apostolov A. 1991. - Copépodes Harpacticoides cavernicoles de Bulgarie. 2. Deux nouveaux rẹprésentants du genre Siygoelaphoidella Apostolov, 1985 du nord-ouest de la Bulgarie. Annis Limnol. 27 (1) : 25.35.

Lang K. 1948. - Monographie der Harpacticiden. 2 vol. Lund : $1682 \mathrm{p}$. 